\title{
A News Image Captioning Approach Based on Multi-Modal Pointer-Generator Network
}

\author{
Jingqiang Chen and Hai Zhuge \\ School of Computer Science, Nanjing University of Posts and Telecommunications, China \\ Aston University, Birmingham, UK
}

\section{SUMMARY}

News image captioning aims to generate captions or descriptions for news images automatically, serving as draft captions for creating news image captions manually. News image captions are different from generic captions as news image captions contain more detailed information such as entity names and events. Therefore, both images on news and the accompanying text are the source of generating caption of news image. Pointer-generator Network is a neural method defined for text summarization. This paper proposes the Multi-modal Pointer-generation Network by incorporating visual information into the original network for news image captioning. The multi-modal attention mechanism is proposed by splitting attention into visual attention paid to the image and textual attention paid to the text. The multi-modal pointer mechanism is proposed by using both textual attention and visual attention to compute pointer distributions, where visual attention is first transformed into textual attention via the Word-Image Relationships. The multi-modal coverage mechanism is defined to reduce repetitions of attentions or repetitions of pointer distributions. Experiments on the DailyMail test dataset and the out-of-domain $B B C$ test dataset show that the proposed model outperforms the original pointer-generator network, the generic image captioning method, the extractive news image captioning method, and the $L D A$-based method according BLEU, METEOR and ROUGL-L evaluations. Experiments also show that the proposed multi-modal coverage mechanisms can improve the model, and that transforming visual attention to pointer distributions can improve the model.

KEYWORDS: Pointer-generator network, multi-modal summarization, text summarization, image captioning

E-mail correspondence: h.zhuge@aston.ac.uk 


\section{INTRODUCTION}

News image captioning aims to generate captions or descriptions for news images automatically. News image captioning can provide draft captions for news images which is significant in applications because there are great number of news images on the Internet and to create manual captions is a time- and labor-consuming task. Many online news sites such as $C N N$, DailyMail, BBC, Yahoo! publish images with their stories and even provide photo feeds related to current events. These news sites are a good resource for multimedia files containing information in form of videos, images and natural language texts, and thus provide captioned news images corpora for supervised machine-learning based captioning approaches.

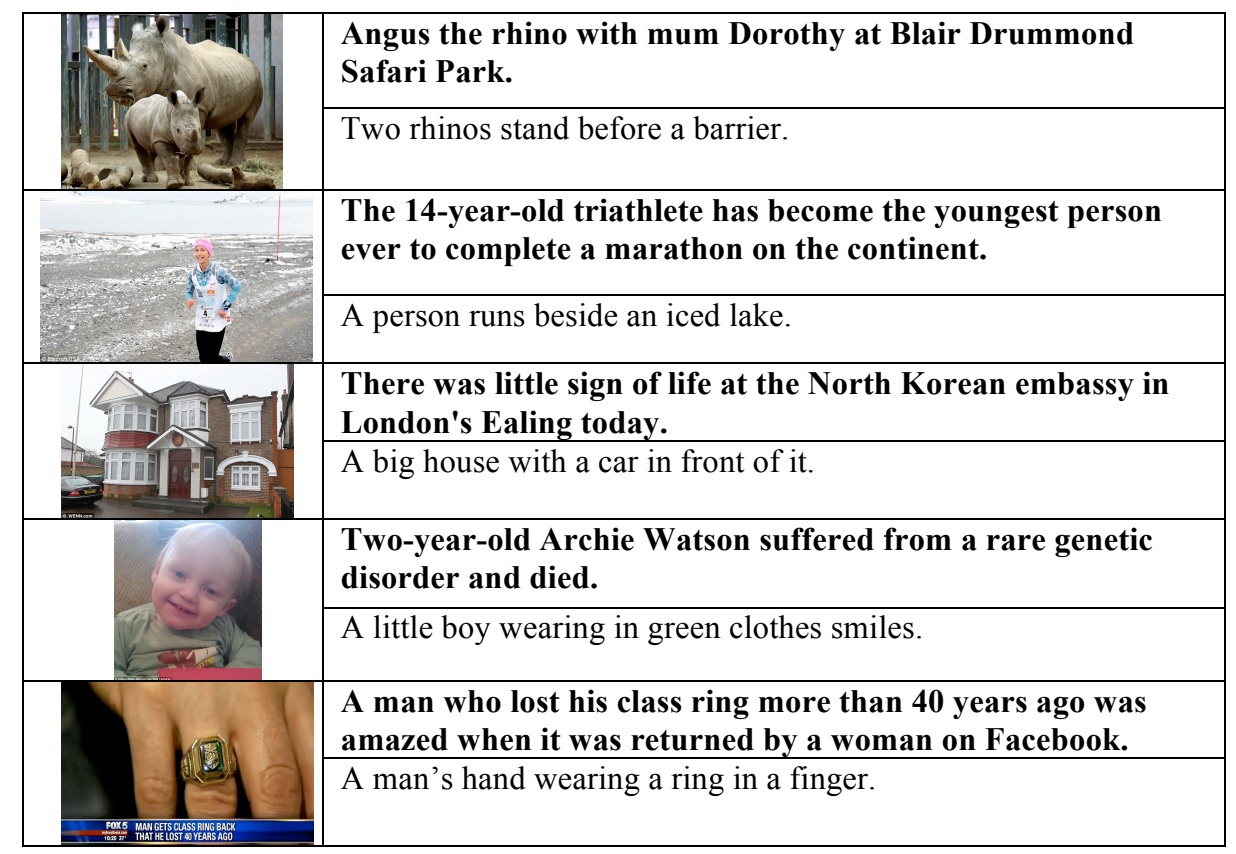

Figure 1. Five news images taken from DailyMail, each with its original caption in the top right and a generic caption in the bottom right.

News image captioning is different from generic image captioning mainly in that news images are related to the texts of news and therefore captions of news images should contain information of the surrounding texts of news images. The caption of a news image often reflects the specific event reported in the news. While generic image captioning generates generic image captions which only contain generic information of images and cannot reflect specific information in news. This is mainly because generic image captioning focuses on the image itself which is the only information that can be used for generating the caption. 
Generic image captioning does not take into consideration the related or surrounding text of the image because not all images have related texts as news images do.

Figure 1 shows the news image captions and generic image captions for five news images taken from DailyMail to demonstrate the difference between the two types of captions. One difference is that news image captions usually contain more detail information than generic captions. For example, the generic caption of the first image is "Two rhinos stand before a barrier", while the original news image caption gives the names of the two rhinos, the relationships between them, and the name of the standing place Safari Park. The detail information is contained in the text of the news. The other difference is that news image captions often contain information on specific events reported in news. Taking the fourth image for example, its original caption shows that the boy suffered from a disaster and died while the generic caption does not contain this information. Another example is about the second image. The original caption contains the event that a young person completes a marathon on the continent while the generic caption only shows a person runs beside an lake shown in the image. It is hard to deduce the detailed information and the event information merely from images because they are usually contained in the text of news.

Therefore, to generate news image captions shown in Figure 1, both the image and the text of the news should be taken into consideration. Recent image captioning techniques focus on generic image caption generation and mainly utilize image information to generate captions (Vinyals et al., 2015; Xu et at., 2015; Liu et al., 2017; You et al., 2016; Zhao et al., 2019), and do not make full use of the accompanying text of the image because not all images have accompanying texts. On contrary, neural text summarization methods focus on text information to generate summaries (See et al., 2017), while neglecting image information.

This paper proposes the Multi-modal Pointer-generator Network for news image captioning by incorporating visual information into the state-of-the-art text summarization model Pointer-generator Network (See et al., 2017; Bahdanau et al., 2014; Luong et al., 2015). The proposed network consists of a multi-modal generator and a multi-modal pointer mechanism. At each decoding step, the model splits the attention into textual attention paid to text and visual attention paid to the image. The generator computes the vocabulary distributions from the text and the image. The pointer mechanism transforms visual attention into textual attention through the Word-VisualPart relationships which are defined between each source word and each visual part of the source image. The pointer distributions are computed by summing up the transformed visual attention and the visual attention. Tow multi-modal coverage mechanisms are proposed to alleviate the repetition problems: one is defined over attention and the other is defined over pointer distributions. The model is trained on the corpora constructed by collecting the first images of the news in the original DailyMail corpora (Cheng and Lapata, 2016).

The main contributions of this paper are as follows:

1) The multi-modal pointer-generator network is proposed for news image captioning by incorporating visual information into the original pointer-generator network. 
2) The multi-modal pointer mechanism is proposed in the network to utilize both textual attention and visual attention to compute pointer distributions by first transforming visual attention into textual attention based on the Word-VisualPart relationships modeled by an attention mechanism.

3) Two multi-modal coverage mechanisms are defined in the model by reducing the repetitions of attentions or the repetitions of pointer distributions.

Experiments carried out on the DailyMail test dataset and the out-of-domain $B B C$ test dataset show that the proposed multi-modal pointer-generator network outperforms the original pointer-generator network, the generic image captioning method, the $L D A$-based method, and the neural extractive method according to BLEU, METEOR and ROUGE-L measures. Experiments also show that incorporating visual attention into the pointer mechanism can improve the proposed model, and that the two multi-modal coverage mechanisms can also improve the proposed model.

\section{RELATED WORK}

News image captioning is tightly related to but is also different from text summarization and generic image captioning. The former generates short summaries from texts, and the latter generates captions from images.

Text summarization can be used to generate news image captions by summarizing news texts. Recent neural text summarization models are based on the attentional Encoder-Decoder model which is first proposed in machine translation area to generate text and to align the original text and the translated text (See et al., 2017; Bahdanau et al., 2014; Luong et al., 2015). The pointer-generator network is proposed to alleviate the Out-of-Vocabulary problem of the encoder-decoder model (See et al., 2017; Zeng et al., 2016) by copying words from source texts. The model is applied to sentence summarization by considering the neural language model and the attention model when generating next words (Rush et al., 2015). A neural document summarization model is proposed by extracting sentences and words (Cheng and Lapata, 2016), where sentences are extracted by computing the probability of sentences belonging to the summary based on an $R N N$ model, and word are extracted from the original document based on an attentional decoder. An $R N N$-based extractive summarization named SummaRuNNer, which treats summarization as a sentence classification problem and applies a logistic classifier using coverage features and redundancy features computed based on the $R N N$ model is proposed (Nallapati et al., 2017). Neural multi-document summarization is also studied. The hierarchical transformer is proposed for multi-document summarization by adding inter-paragraph attention into the transformer (Vaswani et al., 2017; Liu and Lapata, 2019). The MMR (Carbonell and Goldstein, 1998) is incorporated into the pointer-generator network for multi-document summarization (Fabbri et al., 2019; Lebanoff et al., 2018). Another category of summarization is based on discovery of features in texts and between texts and images (Zhuge, 2016). 
Most neural image captioning models are combination of the convolution neural network $(C N N)$ and the recurrent neural network $(R N N)$ (Mao et al., 2014; Jia et al., 2015; Wang et al., 2016; Liu et al., 2017; Wang et al., 2018; Venugopalan et al., 2017; Ren et al., 2017; Chen et al., 2018; Wang and Chan, 2018; Simonyan and Zisserman, 2014). The CNN models such as VGGNet (Ren et al., 2017), AlexNet (Krizhevsky et al., 2012), GoogleNet (Szegedy et al., 2015), ResNet (He et al., 2016) and SENets (Hu et al., 2018) are used to encode images by extracting the last full-connected layers or convolution layers as the vector representations of images. The $R N N$ models are used to encode and decode captions. The first deep learning-based image captioning model is proposed in (Luong et al., 2015) by using a multi-modal recurrent neural network guided by image information. The Encoder-Decoder model was further applied to image captioning by encoding image using the $C N N$ model which is fed into the $R N N$ decoder to generate words one by one (Vinyals et al., 2017). The image encoding is used only once in the decoder as the initial input, and the previously generated word is used as the only input to the next decoding steps to generate the next words. The model is extended in (Xu et al., 2015) by adding the attention mechanism (Bahdanau et al., 2014; Luong et al., 2015) where the image is split into multiple parts which are taken as the initial input of the decoder and are attended to compute the context in each decoding step. The correctness of attention mechanism was further studied in (You et al., 2016) and a supervised attention mechanism is proposed, and the results show that the alignments created by the attention mechanisms are in high accordance with manual alignments. The semantic attention mechanism is proposed in (Liu et al., 2017) which makes use of image tags as additional information and attends image tags during decoding. A multi-modal Transformer-based model is proposed in (Zhao et al., 2019) to ingest both entity labels and image features for generic image captioning. Image can also be represented as a collection of objects which are encoded by the $R N N$ model, and then the attentional mechanism is applied to the objects in the decoder (Liu et al., 2017; Wang et al., 2018; Venugopalan et al., 2017). A hierarchical LSTMs with adaptive attention for visual captioning was proposed (Gao, et al., 2019). Other advances in image captioning are based on reinforcement learning (Ren et al., 2017) and the generative adversarial nets (Chen et al., 2018).

News image captioning is different from generic image captioning and text summarization. The image information and the text information should be both taken into consideration to generate captions for news images. An early study on news image captioning is based on the probabilistic model (Feng and Lapata, 2013). It treats the image and text as a collection of visual words and textual words, and applies the $L D A$ model to compute a mixture model of topics and words, based on which the extractive caption generation model and abstractive caption generation model are proposed (Blei et al., 2003). A neural extractive news image captioning method is proposed in (Batra et al., 2018). The method encoded the ordering embeddings of images and texts with LSTM into a context vector to summarize the multi-modal document, and uses as the object function the cross entropy between captions and the context vector. The sentences are extracted as captions based on the cosine similarity with the context vector. Other similar work is text-image 
summarization (Chen and Zhuge, 2018; Chen and Zhuge, 2019), which creates multi-modal summaries with images aligned with sentences.

This work is different from generic image captioning and text summarization in that both the image and the accompanying text are utilized to generate news image captions. The multi-modal pointer-generator network is proposed by incorporating image information into the original pointer-generator network.

\section{BACKGROUND: NEURAL SUMMARIZATION AND IMAGE CAPTIONING}

The encoder-decoder architecture has become the de facto standard for neural abstractive text summarization (Rush et al., 2015) and neural image captioning (Xu et al., 2015). The encoder for text summarization is often a bi-directional LSTM (Sundermeyer et al., 2012) or $G R U$ (Cho et al., 2014) converting the input text to a set of hidden states $\left\{h^{e T}{ }_{i}\right\}$, one for each input word, indexed by $i$. The encoder for image captioning is often a $C N N$-based model pre-trained in ImageNet (Krizhevsky et al., 2012) converting the image into visual vector representations $\left\{h^{e V}\right\}$ by extracting the last full-connection layer or the last convolution layer. The decoder of both neural text summarization and neural image captioning is a unidirectional $R N N$ ( $L S T M$ or GRU) that generates a summary or a caption by predicting one word at a time. The decoder hidden states are represented by $\left\{h_{i}^{d}\right\}$, indexed by $t$. For text summarization, the input text is treated as a sequence of words, and the model is expected to capture the source syntax inherently. For image captioning, the input image is treated as a set of visual parts, and the model is expected to capture relationship between the image and the caption.

$$
\begin{aligned}
& a_{t, i}=v^{T} \tanh \left(W^{a}\left[h_{t}^{d}\left\|h_{i}^{e}\right\| \operatorname{cov}_{t, i}\right]+b^{a}\right) \\
& \alpha_{t, i}=\operatorname{soft} \max \left(a_{t, i}\right) \\
& \operatorname{cov}_{t, i}=\sum_{t^{\prime}=0}^{t-1} \alpha_{t, i}
\end{aligned}
$$

The attention mechanism is proposed to improve the encoder-decoder model. Equations (1) to (3) are the equations for attention calculations in each decoding step used in Pointer-Generator Network (See et al., 2017). The attention weight $\alpha_{t, i}$ measure the importance the input words or visual parts of the image to generating each output word (Equation (1) and (2)), calculated by measuring the strength of interaction between the decoder hidden stated $h_{t}{ }^{d}$, the encoder hidden state $h^{e}$ (the text encoder $h^{e T}$ or the image encoder $h^{e V}$ ), and the cumulative attention $\bar{a}_{t, i}$ (Equation (3)). The notation $\operatorname{cov}_{t, i}$ denotes the degree of coverage which the $i^{\text {th }}$ input word of the source text or input visual part of the source image receives for the first decoding step to the $i-1^{\text {th }}$ step. A large value of $\operatorname{cov}_{t, i}$ indicates the $i^{\text {th }}$ input word or visual part has been used prior to time $t$ and it is unlikely to be used again for generating the $t^{\text {th }}$ output word. 


$$
\begin{gathered}
c_{t}=\sum_{i} \alpha_{t, i} h_{i}^{e} \\
P_{\text {vocab }}(w)=\operatorname{soft} \max \left(W^{y}\left[h_{t}^{d} \| c_{t}\right]+b^{y}\right)
\end{gathered}
$$

The context vector $c_{t}$ is computed by Equation (4) to summarize the semantic meaning of the input, which is a weighted summation of the encoder hidden states. The vocabulary probability $P^{v o c a b}(w)$ which measures the probability of a vocabulary word $w$ being selected as the $t^{\text {th }}$ output word are then computed by using the context vector and the decoder $\left(h^{d}{ }_{t} \| c_{t}\right)$ in Equation (5).

$$
\begin{gathered}
P_{\text {gen }}=\operatorname{sigmoid}\left(w^{z}\left[h_{t}^{d}\left\|c_{t}\right\| y_{t-1}\right]+b^{z}\right) \\
P(w)=p_{\text {gen }} P_{\text {vocab }}(w)+\left(1-p_{\text {gen }}\right) \sum_{i: w_{i}=w} \alpha_{t, i}(7)
\end{gathered}
$$

Especially, to deal with the out-of-vocabulary problem $(\mathrm{OOV})$ of neural text summarization, a copy mechanism is provided in Pointer-Generator Network by adding a "switch" which is estimated $\left(p_{g e n} \in[0,1]\right)$ to indicate whether the system has chosen to select a word from the vocabulary or to copy a word from the source text (Equation (6)). The switch is calculated using a feed forward layer with the sigmoid activation over [ $\left.h_{t}^{d}\left\|c_{t}\right\| y_{t-1}\right]$, where $y_{t-1}$ is the embedding of the output word at the $t-1^{\text {th }}$ decoding step. Equation (7) computes the final probability $P(w)$ for the word w which is a weighted combination of the vocabulary probability and the copy probability. The attention weights of the word $w$ is used to calculate the copy probability $\sum_{i: w_{i}=w} \alpha_{t, i}$. If the word $w$ appears once or more times in the source text, the copy probability is the summation of its occurrences. For image captioning, this type of pointer mechanism is not applicable though it also has $O O V$ problems.

$$
\begin{gathered}
\operatorname{cov} \text { loss }_{t}=\sum_{i} \min \left(\alpha_{t, i}, \operatorname{cov}_{t, i}\right) \\
\text { loss }_{t}=-P\left(w^{*}\right)+\lambda \operatorname{cov} \operatorname{loss}_{t}
\end{gathered}
$$

The objective function for training Pointer-Generator Network consists of two parts (Equation (8) and (9)): the primary negative log-likelihood loss function $\left({ }^{-P\left(w^{*}\right)}\right)$ and the coverage loss $\left(\sum_{i} \min \left(\alpha_{t, i}, \operatorname{cov}_{t, i}\right)\right.$ ). The coverage loss is bounded and is always less than 1 . The coverage loss is used to penalize repeatedly attending to the same input words of the source text or the same visual parts of the source image, and thus can alleviate the word or phrase repetition problem. This type of coverage loss is initially defined for neural text summarization (See et al., 2017), and is also applicable for image captioning.

For text summarization, the model can be trained on text summarization data containing a large collection of news articles paired with summaries (See et al., 2017). For generic image captioning, the model can be trained on image captioning data containing a large collection of images paired with captions (Vinyals et al., 2017; Xu et al., 2015). The inputs of text summarization and image captioning are single-modal data, either texts or images. 
However, we wish for the model to be applicable in news image captioning, the inputs of which are multi-modal data containing both texts and images. This brings up two issues. First, the parameters (in Equations (1) to (8)) of the models are ineffective on modeling the relationships between the source text and the source image of news image captioning. As with captions, the accompanying news texts have tight relationships with the news images and the words in news texts can be well aligned with visual parts or objects in news images. Humans are good at discovering these relationships and alignments, and use them to generate high-quality captions. This inspires us to make the encoder-decoder be able to mine the relations and alignments which will also render the model to generate better captions for news images. Second, the attention mechanism for news image captioning will pay attentions to both words of source texts and visual parts of source images. This will lead to the attention distribution problem and the attention redundancy problem between the two modalities. It needs well-decided how much attention the text receives and how much attention the image receives. We conjecture that well attention distribution between the text and the image will also benefit new image caption generation. The attention distribution can be affected by the above mentioned relationships between the text and the image. In the following section, we present our adaptation method of the multi-modal pointer-generator network for news image captioning.

\section{MULTI-MODAL POINTER-GENERATOR NETWORK}

\subsection{Network Architecture}

Figure 2 shows the framework of the model. The proposed multi-modal pointer-generator network is an extension of the original pointer-generator network for news image captioning. The inputs of the model are a news image and its accompanying news text, so the model consists of textual parts and visual parts which are combined in the model to figure out the final caption word distributions for caption generation. The red-colored parts in Figure 2 are the extended new parts in contrast to the original pointer-generator network. As with the original pointer-generator network, the proposed model consists of four parts: the encoders, the decoder, the attention mechanism, and the pointer mechanism. 


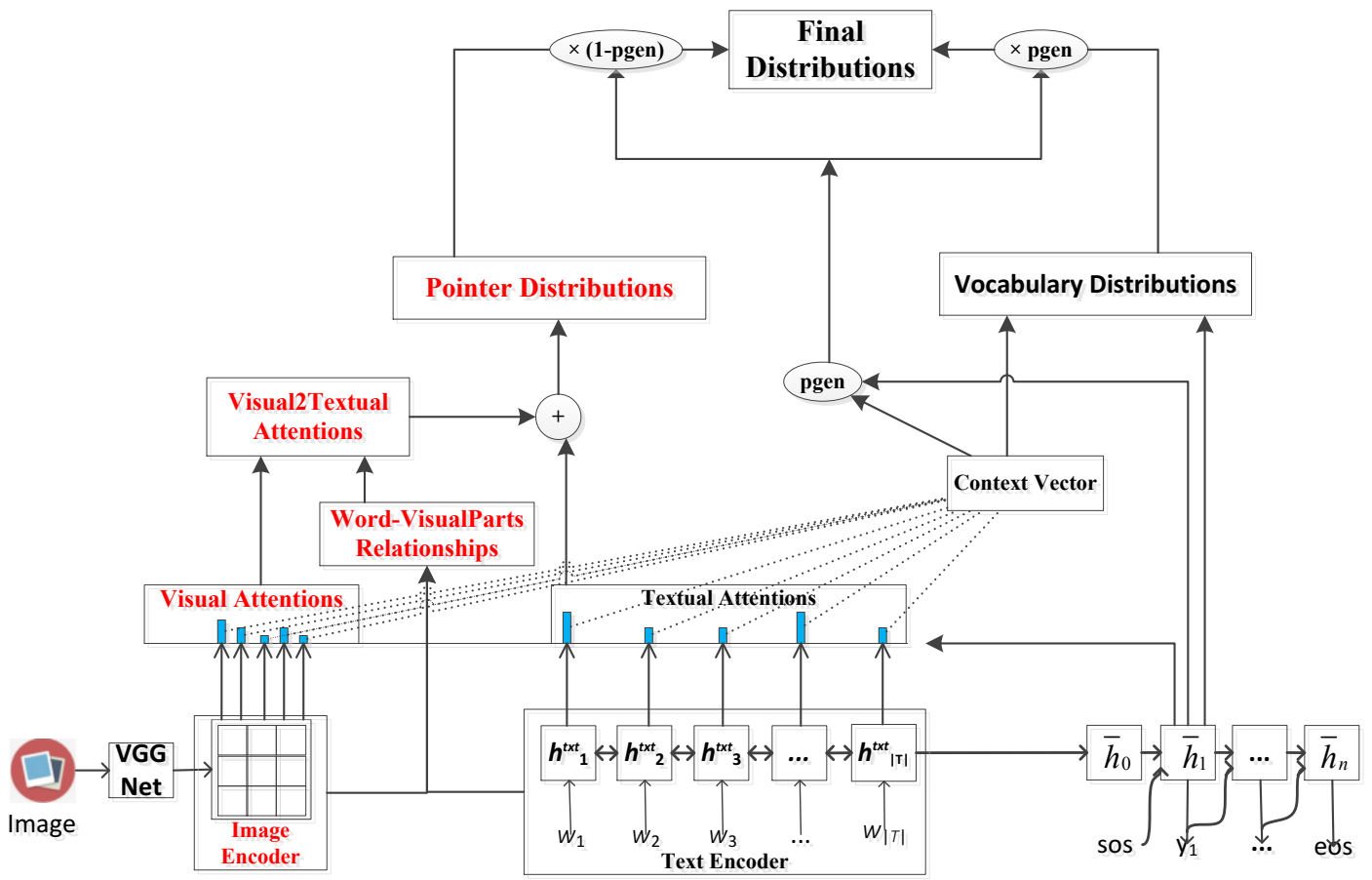

Figure 2. The framework of the proposed multi-modal pointer-generator network.

Encoders. The textual encoder employs the recurrent neural network $(R N N)$ to encode the accompanying new text into vector representations. The visual encoder employs the state-of-the-art convolution neural network Oxford VGGNet (Simonyan and Zisserman, 2014) to extract vector representations for images.

The bi-directional $R N N$ model is used as the text encoder to encode the accompanying text $T$. Equations (10) to (12) are the equations of the bi-directional $R N N$ model, where $x^{i}$ represents the word embedding of the $i^{\text {th }}$ word of $T$. The gated recurrent unit (GRU) (Cho et al., 2014) is adopted as the $R N N$ cell in our method because it is as efficient and effective as LSTM while less time-consuming.

$$
\begin{aligned}
& \vec{h}^{e T}{ }_{i}=G R U^{\overrightarrow{e T}}\left(\vec{h}^{e T}{ }_{i}, x_{i}\right)(10) \\
& \overleftarrow{h}^{e T}{ }_{i}=G R U^{\overleftarrow{e T}}\left({ }^{e T}{ }_{i}, x_{i}\right) \text { (11) } \\
& h^{e T}=\left[\vec{h}_{|T|}^{e T} \| \tilde{h}_{\|}^{e T}\right]
\end{aligned}
$$

The Oxford VGGNet is used as the visual encoder to encode the image into vector representations. VGGNet is initially used for image classification. It consists of several convolution layers each followed by a pooling layer and the last fully-connected layers. The 
last convolution layer splits the image into $14 \times 14$ visual parts denoted as $\left\{v_{1}, v_{2}, \ldots, v_{196}\right\}$ each of which encoded into a 512-dimensional vector representation, and has been proved suitable for image captioning and for the attention mechanisms to attend (Xu et al., 2015). A non-linear tanh transformation is applied to $v_{i}$ to get the final encoding of each visual part, Equation (13).

$$
h_{i}^{e I}=\tanh \left(M^{e I} \cdot v_{i}+b^{e I}\right)
$$

Decoder. The $R N N$-based decoder is used to generate words one by one in our method. Equations (14) to (15) are the equations for the decoder. Equation (14) computes the initial hidden state of the decoder from the encoding of the accompanying text. Here only the text encoding is used in initial state computation. Different from that of the original pointer-generator network, the context $c_{t}$ in Equation (15) is multi-modal, which is the summation of the textual encodings and visual encodings weighted by the multi-modal attentions. The vocabulary distribution of the words is also computed by Equation (5) as in the original pointer-generator network.

$$
\begin{aligned}
& h_{0}^{d}=\tanh \left(W^{d 0} \times h_{-1}^{e T}+b^{d 0}\right) \\
& h_{t}^{d}=G R U^{d}\left(h_{t-1}^{d}, y_{t-1} \| c_{t}\right)
\end{aligned}
$$

Multi-Modal Attentions. The attention is paid to both the image and the accompanying text for news image captioning. The attentions paid to the image are named by visual attentions, and the attentions paid to the text are named by textual attentions. As in the original pointer-generator network, Equation (1) is used to compute the un-normalized textual attention $a_{t, i}^{T}$ for each word encoding $h^{e T}{ }_{i}$, and to compute the un-normalized visual attention $a_{t, j}^{V}$ for each visual part encoding $h^{e V}$. The normalized textual attentions and visual attentions are computed by Equation (16) and (17). So the multi-modal context $c_{i}$ is computed by Equation (18).

$$
\begin{aligned}
\alpha_{t, i}^{T} & =\frac{\exp \left(a_{t, i}^{T}\right)}{\sum_{i} \exp \left(a_{t, i}^{T}\right)+\sum_{j} \exp \left(a_{t, j}^{V}\right)} \\
\alpha_{t, j}^{V} & =\frac{\exp \left(a_{t, j}^{V}\right)}{\sum_{i} \exp \left(a_{t, i}^{T}\right)+\sum_{j} \exp \left(a_{t, j}^{V}\right)} \\
c_{t} & =\sum_{i} \alpha_{t, i}^{T} h_{i}^{e T}+\sum_{j} \alpha_{t, j}^{V} h_{j}^{e V}
\end{aligned}
$$

Final Distributions. As with the original pointer-generator network, the final distributions of caption words at the $t^{\text {th }}$ decoding step are summation of the vocabulary distributions and the pointer distributions (Equation (6) and (7)). The pointer distributions in the original pointer-generator are only determined by the textual attentions. However, the pointer distributions in the multi-modal pointer-generator are determined by both the textual 
attentions and the visual attentions. The definition of the multi-modal pointer mechanism will be discussed in the following subsections.

Training. The loss function $L$ of our news image captioning model is the negative log likelihood of generating captions over the training set as defined in Equation (19) and (20), where $\left\langle I, T, Y>\right.$ is an image-text-caption tuple of the training set, and $Y=\left[y_{1}, y_{2}, \ldots, y_{\mid Y]}\right]$ is the word sequences of the caption including the start token $\langle\operatorname{sos}\rangle$ and the end token $\langle e o s\rangle$. In Equation (20), $\log \left(P\left(y_{t} \mid\left\{y_{1}, \ldots, y_{t-1}\right\}, c ; \theta\right)\right)$ is modeled by the proposed news image captioning model. The Adam (Kingma and $\mathrm{Ba}, 2014$ ) gradient-based optimization method is adopted to optimize the model parameters.

$$
\begin{gathered}
L=\sum_{<I, T, Y>\in \text { TrainingSet }}-\log P(Y \mid I, T) \\
\log P(Y \mid I, T)=\sum_{t=1}^{|Y|} \log P\left(y_{t} \mid\left\{y_{1}, \ldots, y_{t-1}\right\}, c ; \theta\right)
\end{gathered}
$$

Inferring. For inferring, the beam search algorithm is adopted at test time. The beam width is set as 5 in the experiments. The inferring stops when it generates the end token $<e o s>$.

\subsection{Calculations of multi-modal pointer distributions}

The attention is split to textual attention paid to the source text and visual attention paid to the source image. The textual attention can be straightforwardly used as pointer distributions over the source words as in the original pointer-generator network. While the visual attentions can also influence pointer distributions because an image has relationships with its accompanying text and visual parts of the image can be aligned with the words in the text. Therefore, visual attentions can be transformed into textual attentions and then used to calculate pointer distributions.

The red-colored parts in Figure 2 are especially for calculations of multi-modal pointer distributions. The visual attentions are first transformed to pointer distributions through the Word-VisualPart Relationships (Equation (21)), and are then combined with textual attentions to get the whole pointer distributions (Equation (22)).

The Word-VisualPart relationships are calculated in Equation (21) and (22) by applying an attention mechanism between the hidden state $h^{e T}{ }_{i}$ of each source word $i$ and the hidden state $h^{e I}{ }_{j}$ of each visual part $j$. Here the hidden state $h_{j}^{e I}$ is used as the query and the hidden state $h^{e T}$ is used as the key. In Equation (19), the matrix ${ }^{M^{d^{T} x d^{d}}}$ is the added parameters to compute the attention between $h^{e I}$ and $h_{i}^{e T}$, where $d^{e T}$ is the dimension size of $h^{e T}$ and $d^{e I}$ is the dimension size of $h^{e I}$. In the proposed model, $d^{e T}$ is set as the same with $d^{e I}$. Equation (22) normalizes the values by using the softmax function to make $\sum_{i} r_{j, i}=1$.

$$
\bar{r}_{j, i}=\tanh \left(h_{j}^{e l} M^{d^{e l} \times d^{e T}}\left(h_{i}^{e T}\right)^{T}\right)
$$




$$
r_{j, i}=\operatorname{soft} \max \left(r_{j, i}\right)
$$

Next, the visual attentions are transformed into textual attentions (named after Visual2Textual attentions in Figure 2 via the Word-VisualPart relationships. Equation (23) is for calculations of Visual2Textual attentions. At the $t^{\text {th }}$ decoding step, the Visual2Textual attention that each source word $i$ receives is the summation of the transformed attentions that each visual part $j$ distributes to the word $i$. The portion of the transformed attention is determined by the relationship $r_{j, i}$.

$$
\alpha_{t, i}^{V 2 T}=\sum_{j} \alpha_{t, j} r_{j, i}
$$

Finally, the multi-modal pointer distribution of the word $w$ is calculated by summing up the textual attention and the Visual2Textual attention. Equation (24) is the equation for calculations of the pointer distribution. The final distribution is calculated by adding up the vocabulary distribution and the multi-modal pointer distribution as in Equation (7).

$$
P_{p t r}(w)=\sum_{i: w_{i}=w} \alpha_{t, i}+\alpha_{t, i}^{V 2 T}
$$

\subsection{Multi-modal coverage mechanism}

The repetition problem also exists in the visual attentions as well as in the textual attentions of the original pointer-generator network, so the multi-modal coverage mechanism is defined by taking visual attentions into consideration. The aim of the coverage mechanism is to reduce repetitions over attentions as well as repetitions over pointer distributions. However, pointer distributions over source words are not equivalent to textual attentions in the multi-modal pointer-generator network. Therefore, two methods for the multi-modal coverage mechanism are proposed in the following.

The first method is defined over attentions, and uses visual attentions the same way with textual attentions to define the coverage vector as in Equation (3) and to define the coverage loss as in Equation (8). This is a straightforward method to avoid repetitions over textual attentions and visual attentions, and repetitions over pointer distributions can be indirectly reduced.

The second method is defined over multi-modal pointer distributions. This method transforms visual attentions into textual attentions and controls the repetitions in the transformed attentions. Equation (25) and (26) is the equations for calculations of the coverage vector and the coverage loss for this method, both of which are computed over the multi-modal pointer distributions. The repetitions over attentions can be indirectly reduced through controlling repetitions over the multi-modal pointer distributions.

$$
\begin{gathered}
\operatorname{cov}_{t, i}=\sum_{t^{\prime}=0}^{t-1} \alpha_{t, i}+\alpha_{t, i}^{V 2 T} \\
\operatorname{cov} \text { loss }_{t}=\sum_{i \in T} \min \left(\alpha_{t, i}, \operatorname{cov}_{t, i}\right)
\end{gathered}
$$

Which calculation method for the multi-modal coverage mechanism performs better will be discussed in the following experiments. 


\section{EXPERIMENTS}

\subsection{Data}

Two news image captioning datasets are provided: one large-scale dataset for training and testing, and the other small-scale dataset only for testing.

The large-scale training and testing corpora are constructed from the DailyMail news corpora. The standard DailyMail corpora are the widely used corpora originally built in (Hermann et al., 2015) by collecting news stories from the DailyMail news websites for question answering and document summarization. There are about $210 \mathrm{~K} \mathrm{html-formatted}$ news documents provided in the original DailyMail news corpora. Each html-formatted news document contains one or more image-image pairs. To create news image-caption-text dataset, we extract and collect the first image-caption pair and the main text of each news document by parsing the html-formatted documents. The created news image captioning corpora are split into train, dev, and test dataset as in the original DailyMail corpora. The split and statistics of the created DailyMail news image captioning corpora are shown in Table 1.

Table 1. The split and statistics of the DailyMail news image captioning corpora

\begin{tabular}{ll}
\hline \hline \multicolumn{1}{c}{ Description } & \multicolumn{1}{c}{ Value } \\
\hline Size of training dataset & 187900 \\
Size of dev dataset & 11410 \\
Size of testing dataset & 9814 \\
Average number of words in news texts & 663.88 \\
Average number of words in news & 26.78 \\
captions & \\
\hline \hline
\end{tabular}

Table 2. The split and statistics of the $B B C$ corpora

\begin{tabular}{ll}
\hline \hline \multicolumn{1}{c}{ Description } & \multicolumn{1}{c}{ Value } \\
\hline $\begin{array}{l}\text { Size of testing dataset } \\
\text { Average number of words in news }\end{array}$ & 240 \\
texts & 422.01 \\
$\begin{array}{l}\text { Average number of words in news } \\
\text { captions }\end{array}$ & 9.59 \\
\hline \hline
\end{tabular}


The other corpora are originally provided in (Fend and Lapata, 2013) for probabilistic news image captioning. The corpora are collected from the $B B C$ news website, and contain 3361 image-caption-text tuples in all, 240 of which are for testing. Due to such a small size, the corpora are only used to test the model trained with the DailyMail corpora introduced above. The statistics of the $B B C$ corpora is shown in Table 2 .

During training and at the test time, the news texts are truncated to 400 tokens, the ground-truth summaries are truncated to 100 tokens.

\subsection{Implementation}

The texts of the corpora are preprocessed by tokenizing the text and replacing the digits with the $<N U M>$ token. The 40k most frequent words in the corpora are kept and other words are replaced with the $\langle O O V\rangle$ token. The word embeddings are initialized with Google's word2vec tools (Mikolov et al., 2013) trained in the whole text of DailyMail and BBC corpora. The dimension of the word embeddings are set as 128 .

The images are encoded by extracting the $14 \times 14 \times 512$ conv5_4 layer of the 19-layer VGGNet (Simonyan and Zisserman, 2014) pre-trained on ImageNet as the vector representation of images, where $14 \times 14$ is the number of visual parts and 512 is the dimension of each visual part.

The proposed model is implemented based on See et al. (See et al., 2017)'s Pointer-Generator Network written with Tensorflow. The dimension of the hidden state of the $R N N$ decoder is 512. The beam width is set as 5. The parameters of Adam are set to those provided in (Kingma and $\mathrm{Ba}, 2014$ ). The batch size is set to 12. Gradient clipping is employed to regularize our models. All models are trained on a GTX-1080 TI GPU card for 400,000 steps. The best checkpoint is selected based on performance on the validation set and the results on the test set are reported.

\subsection{Comparisons with existing methods}

The frequently used $B L E U$ metric (Papineni et al., 2002) which is the standard in image caption generation research is adopted. $B L E U$ without a brevity penalty is reported. There has been, however, criticism of $B L E U$, so another common metric METEOR (Denkowski and Lavie, 2014) is reported and compared whenever possible. The widely used summarization evaluation metric ROUGE (Lin, 2014) is also adopted to evaluate the generated captions.

The proposed method in this paper is compared with five existing methods on the DailyMail corpora and the $B B C$ test dataset. The evaluation results are shown in Table 3 and Table 4. The compared methods are listed and described as follows:

-- MMPtrGen is the proposed multi-modal pointer-generator network without the coverage mechanism. 
-- MMGen is the proposed multi-modal pointer-generator network without the pointer mechanism and the coverage mechanism.

-- PtrGen-T is the original pointer-generator network carried out on the news text without the coverage mechanism.

-- Gen-T is the state-of-the-art generative text summarization method based the attentional encoder-decoder model proposed in (Rush et al., 2015). This method treats the news image captioning problem as the text summarization problem and only uses the news text as the input. The method generates the news image caption by treating the news text as a long sentence and summarizing the text.

-- Gen-I is the state-of-the-art generic image captioning method based on the attentional encoder-decoder model proposed in (Xu et al., 2015). This method only uses the image as the input, splits the image into 196 visual parts encoded with the $C N N$ model, and uses the attentional decoder to generate captions.

-- NNExtr is the neural news image captioning method recently proposed in (Batra et al., 2018). This method uses a neural classification model to score the sentences in the news text and extracts the most relevant sentence as the caption. The method first computes the context vector with LSTM using the ordering embeddings of images and texts, and then trains a sentence classification model using as the object function the cross entropy between captions and the context vector. The sentences are scored according to the cosine similarity with the context vector.

-- LDA-based is the state-of-the-art probabilistic news image captioning method proposed in (Feng and Lapata, 2013), and the results on the $B B C$ test dataset are reproduced in (Batra e tal., 2018) for comparison. The method works as follows. Firstly, textual dictionaries are synthesized by assigning a unique token $i d$ to each word present in any of the articles, and visual dictionary is made by clustering SIFT descriptors into 2,000 different visual words. Secondly, a $L D A$ model is trained with 1,000 topics on the $B B C$ news dataset containing both text and images. Thirdly, extractive summarization is used for surface realization. It has been shown in (Feng and Lapata, 2013) that retrieving sentences based on the Kullback-Leibler divergence between the topic distribution of a sentence and the topic distribution of a news article gives the best results in terms of human evaluation.

According to Table 3, the proposed multi-modal pointer-generator network MMPtrGen gets the highest BLEU scores, the highest METEOR scores, and the highest ROUGE-L $F$-measure scores among the five methods, and the original pointer-generator network gets the second higher scores. The evaluation results of LDA-based and NNExtr are not shown in Table 3 because they are not reported on the DailyMail corpora. MMPtrGen outperforms PtrGen-T, which implies that incorporating visual information into the pointer-generator network can improves the summarization model, and that by considering both news text and image can generate better captions than only considering text on the DailyMail corpora. PtrGen-T gets the second higher scores, which implies that news text is more suitable for news image captioning than news images. Both MMPtrGen and PtrGen-T outperform the corresponding $M M G e n$ and Gen-T, which implies the pointer mechanism plays an important role in the pointer-generator network and can significantly improve the summarization and captioning model. The state-of-the-art generic image captioning method NNattImg performs 
very poorly on the DailyMail corpora, though it performs well on the generic image captioning corpora such as COCO and Flickr. This means that news image captioning is more likely a text summarization problem than an image captioning problem. News image captions contain detail information and event information not contained in images as introduced in Section I. So considering both news text and news images can generates better captions than only considering news images or news text.

Table 3. Comparisons with the existing methods on the DailyMail corpora.

\begin{tabular}{cccc}
\hline \hline Method & BLEU & METEOR & ROUGE-L \\
\hline MMPtrGen & $\mathbf{9 . 6 2}$ & $\mathbf{1 9 . 6 9}$ & $\mathbf{2 7 . 7 0}$ \\
MMGen & 7.24 & 16.68 & 26.07 \\
\hline PtrGen- $T$ & 9.46 & 19.49 & 27.41 \\
Gen- $T$ & 6.95 & 16.49 & 25.40 \\
Gen-I & 0.33 & 4.20 & 10.41 \\
\hline \hline
\end{tabular}

Table 4. Comparisons with the existing methods on the $B B C$ test dataset.

\begin{tabular}{cccc}
\hline \hline Method & BLEU & METEOR & ROUGE-L \\
\hline MMPtrGen & 0.42 & $\mathbf{1 0 . 7 7}$ & $\mathbf{9 . 2 6}$ \\
MMGen & 0.36 & 9.56 & 9.18 \\
\hline PtrGen-T & $\mathbf{0 . 4 8}$ & 10.58 & 9.24 \\
Gen-T & 0.34 & 9.39 & 9.05 \\
Gen-I & 0.08 & 3.00 & 3.85 \\
NNExtr & 0.34 & 6.77 & - \\
LDA-based & 0.30 & 7.06 & - \\
\hline \hline
\end{tabular}

Table 4 shows the scores on the $B B C$ test dataset. Since our models are trained on the DailyMail corpora, the $B B C$ test dataset is the out-of-domain dataset for our models. The evaluation results in Table of MMPtrGen, MMGen, PtrGen, Gen-T, and Gen-I are in accordance with the results in Table 3. MMPtrGen gets the highest METEOR scores and Rouge- $L$ scores, and PtrGen gets the highest BLEU scores. What's new is that the proposed method MMPtrGen outperforms LDA-based and NNExtr which achieve the state-of-the-art performance in the $B B C$ dataset. Only the BLEU scores and METEOR scores of the two baselines are reported in the original paper. The proposed MMPtrGen achieves new state-of-the-art performance in the $B B C$ dataset, though it is trained on the DailyMail corpora. 
In summary, the proposed method NNattSim performs the best and is thus suitable for news image captioning, and incorporating image information into the pointer-generator network does improve the summarization and captioning model.

\subsection{Evaluations of the multi-modal coverage mechanism}

As previously described, the coverage mechanism aims to alleviate the repetition problem of the encoder-decoder model. In the following, the two proposed multi-modal coverage mechanisms are compared to see whether the coverage mechanism will improve the multi-modal pointer-generator network. The evaluation results on the DailyMail corpora and on the $B B C$ test dataset are shown in Table 5 and Table 6.

-- MMPtrGen with COV1 is the proposed multi-modal pointer-generator method with the first multi-modal coverage mechanism defined over attentions.

-- MMPtrGen with COV2 is the proposed multi-modal pointer-generator network with the second multi-modal coverage mechanism defined over the pointer distributions.

According to Table 5, MMPtrGen with COV1 outperforms MMPtrGen on the BLEU score and the METEOR score. MMPtrGen with COV2 outperforms MMPtrGen on all the three scores. This implies that the multi-modal coverage mechanism can improve the multi-modal pointer-generator network in the DailyMail corpora by alleviating the repetition problem. On the other hand, neither of the two multi-modal coverage mechanisms outperforms each other on all the metrics. MMPtrGen with COV1 gets the highest METEOR score, and MMPtrGen with COV2 gets the highest BLUE score and ROUGE-L score. This implies the coverage mechanism defined over attentions and the coverage mechanism defined over the pointer distributions work differently, and both can improve the proposed pointer-generator network.

Table 5. Evaluation results of the multi-modal coverage mechanism on the DailyMail corpora.

\begin{tabular}{lccc}
\hline \hline \multicolumn{1}{c}{ Method } & BLEU & METEOR & ROUGE-L \\
\hline MMPtrGen & 9.62 & 19.69 & 27.70 \\
MMPtrGen with & 9.67 & $\mathbf{2 0 . 0 0}$ & 27.66 \\
COV1 & & & \\
MMPtrGen with & $\mathbf{9 . 6 9}$ & 19.68 & $\mathbf{2 7 . 8 3}$ \\
COV2 & & & \\
\hline \hline
\end{tabular}

Table 6. Evaluation results of the multi-modal coverage mechanism on the $B B C$ corpora. 


\begin{tabular}{cccc}
\hline \hline Method & BLEU & METEOR & ROUGE-L \\
\hline MMPtrGen & 0.42 & $\mathbf{1 0 . 7 7}$ & $\mathbf{9 . 2 6}$ \\
MMPtrGen with & 0.42 & 9.41 & 8.82 \\
COV1 & & & \\
MMPtrGen with & $\mathbf{0 . 4 6}$ & 9.28 & 8.72 \\
COV2 & & & \\
\hline \hline
\end{tabular}

Table 6 shows the evaluation results in the out-of-the-domain $B B C$ test dataset. The two multi-modal coverage mechanisms do not perform as well as in DailyMail test dataset. The performance of the coverage mechanism depends on the corpora. Nevertheless, MMPtrGen with COV2 achieves a higher BLEU score than MMPtrGen does.

In summary, the two multi-modal coverage mechanisms can improve the multi-modal pointer-generator network.

\subsection{Evaluations of the Word-VisualPart relationships}

The Word-VisualPart relationships are the alignments between the source words and the visual parts of the source image, and are used to transform the visual attentions into textual attentions.

In Table 7 and Table 8, MMPtrGen w./o. WVRela is the proposed multi-modal pointer-generator network without the Word-VisualPart relationships. MMPtrGen w./o. WVRela does not transform visual attentions into textual attentions and only uses the original visual attentions as the pointer distributions. According to the two tables, MMPtrGen outperforms MMPtrGen w./o. WVRela, which implies using the Word-VisualPart relationships to transform visual attentions into pointer distributions can improve the multi-modal pointer-generator network.

Table 7. Evaluation results of the Word-VisualPart relationships on the DailyMail corpora

\begin{tabular}{cccc}
\hline \hline Method & BLEU & METEOR & ROUGE-L \\
\hline MMPtrGen & $\mathbf{9 . 6 2}$ & $\mathbf{1 9 . 6 9}$ & $\mathbf{2 7 . 7 0}$ \\
MMPtrGen w./o. & 9.52 & 19.50 & 27.64 \\
WVRela & & & \\
\hline \hline
\end{tabular}

Table 8. Evaluation results of the Word-VisualPart relationships on the $B B C$ corpora.

\begin{tabular}{llll}
\hline \hline Method & BLEU & METEOR & ROUGE-L \\
\hline
\end{tabular}




\begin{tabular}{cccc}
$\begin{array}{c}\text { MMPtrGen } \\
\text { MMPtrGen w./o. }\end{array}$ & $\mathbf{0 . 4 2}$ & $\mathbf{1 0 . 7 7}$ & 9.26 \\
WVRela & 0.39 & 10.75 & $\mathbf{9 . 3 4}$ \\
\hline
\end{tabular}

Table 9. Evaluation of alignments between words and visual parts on the DailyMail corpora.

\begin{tabular}{ccc}
\hline \hline Method & Average Number of Words & Precision \\
\hline MMPtrGen & 7.7413 & $\mathbf{1 1 . 3 3 \%}$ \\
MMPtrGen with COV1 & 7.7945 & $11.25 \%$ \\
MMPtrGen with COV2 & 8.8532 & $11.12 \%$ \\
\hline \hline
\end{tabular}

Moreover, the Word-VisualPart relationships are used to align source words with visual parts as follows: for each visual part $j$, select the most related source word $i$ to align with such that $r_{i, j}$ is the largest. It is a hard and labor-consuming task to manually create ground-truth alignment of source words and visual parts of images. Therefore, the alignments are evaluated by computing the precision of the aligned word set comparing to the caption word set. The assumption is that the well-aligned words are also the keywords in image captions.

Table 9 shows the average number of the aligned words of the images and the precision on the DailyMail corpora. According to the table, there are about 8 words aligned by a news image, about $11.3 \%$ of which appear in the corresponding captions. This implies that the alignments are focused on several words, some of which are caption words. The alignment is not supervised, and can be improved through supervising methods.

\subsection{Discussion of attention distributions over the text and the image}

The above experiments show that the proposed multi-modal pointer-generator network outperforms the original pointer-generator network not very significantly, and that the generic image captioning method performs poorly in news image captioning. To interpret the performance, an additional experiment is carried out to show the average attention distributions on the news texts and the new images as shown in Table 10. Average textual attentions and average visual attentions are computed by making an average over decoding steps and the test datasets. Calculations of the attention distributions in the proposed model are not supervised. 
Table 10. Attention distributions on the DailyMail corpora

\begin{tabular}{ccc}
\hline \hline Method & Average Textual Attention & Average Visual Attention \\
\hline MMPtrGen & $98.132 \%$ & $1.868 \%$ \\
MMPtrGen with & $98.449 \%$ & $1.551 \%$ \\
COV1 & & \\
MMPtrGen with & $99.300 \%$ & $0.700 \%$ \\
COV2 & & \\
\hline \hline
\end{tabular}

According to the table, more than $98 \%$ of the attention is distributed onto the news text, and less than $2 \%$ of attention is distributed to the news image in the proposed multi-modal pointer-generator network. Note that $100 \%$ of the attention is paid to the news text in the original pointer-generator network, and that $100 \%$ of the attention is paid to the news image in the generic image captioning model. Most information in the news image caption is contained in the news text and some information is contained in the news image. Textual attentions play a more important role than visual attentions do for news image captioning. This can partly explain the performance of the proposed multi-modal pointer-generator network, the original pointer-generator network, and the generic image caption method. Although the precision of the discovered Word-VisualPart relationships is not very high, these relationships can help improve the performance because the visual information can be utilized in the pointer mechanism through these relationships. The supervision method can be used to further improve the attention distributions and the Word-VisualPart relationships.

\subsection{A Case Study}

Figure 3 demonstrate the progress and the result of the proposed MMPtrGen method. The vertical axis denotes the percentage of the visual pointer distributions averaged over all the decoding steps. For example, the highest visual pointer distribution of example\#1 is $0.32 \%$. The total visual pointer distributions of the 5 examples are $0.81 \%, 1.62 \%, 0.62 \%, 0.75 \%$ and $2.3 \%$ respectively. The summation of textual pointer distributions and visual pointer distribution over all the words is 1 . According to Figure 3, for the five examples, the pointer distribution consists of about $98 \%$ textual pointer distribution and about $2 \%$ visual pointer distributions, and the top- 9 words gain most of the visual pointer distribution. Textual attention plays a more important role in news image captioning. 


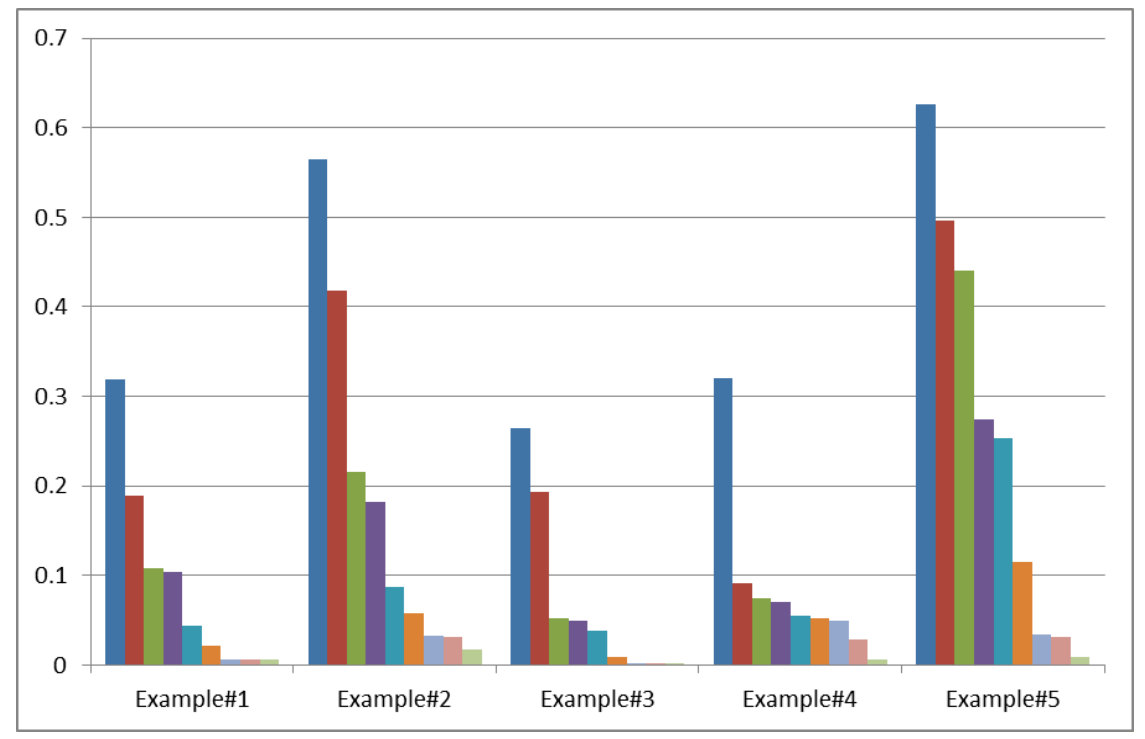

Figure 3. The top-9 average visual pointer distributions over all the decoding steps for the source words of the examples.

Figure 4 shows the generated captions for the five examples. For each image, the ground truth caption is provided on the right top, and the generated caption is provided on the right bottom. As described before, The Arabic numbers in the generated captions are replaced with the token NUM.

The generated captions have high overlaps with the ground truth captions. The generated captions are much better than the generic captions shown in Figure 1, because the generated captions contain detailed information provided in news texts. For example, the caption of the second image contains the information of the age of the triathlete, the marathon which are not shown in the image but contained in the news text. Another example is about the third image, the generated caption contains the information of North Korean embassy in London, which is not contained in the image but is contained in news text. These examples demonstrate that the proposed model can generate satisfactory news image captions.

\begin{tabular}{|l|l|}
\hline & $\begin{array}{l}\text { Angus the rhino with mum Dorothy at Blair Drummond Safari } \\
\text { Park. }\end{array}$ \\
\cline { 2 - 3 } & $\begin{array}{l}\text { the scottish government has joined the fight against the illegal } \\
\text { trade in rhinoceros horn by setting up a dna database. }\end{array}$ \\
\hline & $\begin{array}{l}\text { The 14-year-old triathlete has become the youngest person ever to } \\
\text { complete a marathon on the continent. }\end{array}$ \\
\hline
\end{tabular}




\begin{tabular}{|l|l|}
\hline & $\begin{array}{l}\text { a triathlete who runs marathons across the globe to honour her } \\
\text { cancer victim father has become the youngest person ever to } \\
\text { complete a marathon in antarctica - at just NUM . }\end{array}$ \\
\hline & $\begin{array}{l}\text { There was little sign of life at the North Korean embassy in } \\
\text { London's Ealing today. } \\
\text { the north korean embassy in london was silent today after a } \\
\text { sign appeared on a tree outside the property warning that } \\
\text { loading ' would take place on tuesday }\end{array}$ \\
\hline & $\begin{array}{l}\text { Two-year-old Archie Watson suffered from a rare genetic disorder } \\
\text { and died. }\end{array}$ \\
\hline $\begin{array}{l}\text { tragic: two-year-old archie watson died last monday after } \\
\text { suffering from tay-sachs, which causes deterioration of nerve } \\
\text { cells. }\end{array}$ \\
\hline $\begin{array}{l}\text { A man who lost his class ring more than } 40 \text { years ago was amazed } \\
\text { when it was returned by a woman on Facebook. }\end{array}$ \\
$\begin{array}{l}\text { richard hale, NUM, was reunited with a ring he lost more than } \\
\text { NUM years ago. he was reunited with a ring he lost more than } \\
\text { NUM years ago }\end{array}$ \\
\hline
\end{tabular}

Figure 4. The captions generated by the proposed model for the five pictures in Figure 1. The bottom bold captions are the generated ones.

\section{CONCLUSIONS}

News image captioning task is different from generic image captioning task in that news image captions contain more detailed information such as entity names and events than general image captions do, and detailed information is usually contained in news text but not in news images.

This paper proposes a multi-modal pointer-generator network for news image captioning by incorporating image information into the original pointer-generator network. The proposed network consists of a multi-modal generator which computes vocabulary distributions of the words and a multi-modal pointer which computes pointier distributions of the words. The news image is encoded by $V G G N e t$, and the news text is encoded by the $R N N$ model. The attention is split to textual attention paid to text and visual attention paid to the image to compute the multi-modal context vector in each decoding step. In the multi-modal pointer, visual attention is first transformed into textual attention through the Word-VisualPart relationships modeled by an attention mechanism, and is then added up with the textual attention to compute pointer distributions. Two multi-modal coverage mechanisms are defined by reducing repetitions of multi-modal attentions or by reducing repetitions of pointer distributions. The DailyMail news image captioning corpora are created 
for training and testing by collecting news images, captions, and documents through parsing the html-formatted documents. Another small-sized $B B C$ dataset is used only for testing. Experiments on the two datasets show that the proposed model outperform the original pointer-generator network, the generic image captioning methods, the $L D A$-based news image captioning method, and the neural extractive new image captioning method, which shows that considering both the news image and the news text for generating news image captions is better than considering only new text or image. It is shown by experiments that the model adding visual attention to compute pointer distributions outperform the one not using visual attention as pointer distributions. It is also shown that more than $98 \%$ attention is paid to text and the left is paid to the image, which means that textual attention plays a more important role in the proposed network. Nevertheless, visual attention cannot be neglected because the model considering visual attention outperforms the one without visual attention shown by experiments. Experiments also show the model with the multi-modal coverage mechanisms outperforms the ones without the coverage mechanisms. This paper is extended from our previous work (Chen and Zhuge, 2019).

With the development of smart cameras, images will contain more and more physical features such as time, location, temperature, air quality, and weather where they are taken. Those features indicate the content of images and render the content of text, therefore can help identifying the captions of images. The physical features of images that match the content of text are important for increasing the accuracy and reliability of news. In the future, we will incorporate those features into the implementation of the future interconnection environment (Zhuge, 2005; Zhuge, 2008) for realizing Cyber-Physical-Society and Cyber-Physical-Socio Intelligence CPSI (Zhuge, 2011; Zhuge and Xing, 2012; Zhuge, 2012; Zhuge, 2016; Zhuge, 2020).

\section{ACKNOWLEDGEMENT}

The research was sponsored by the National Natural Science Foundation of China (No.61806101, No.61876048), and the Open Foundation of Key Laboratory of Intelligent Information Processing, ICT, CAS (IIP2019-2). Professor Hai Zhuge is the corresponding author of this paper.

\section{REFERENCES}

1. Vinyals O., Toshev A., Bengio S., Erhan D. "Show and tell: Lessons learned from the 2015 mscoco image captioning challenge." IEEE transactions on pattern analysis and machine intelligence. 2017 Apr 1;39(4):652-63. 
2. Xu K., Ba J., Kiros R., Cho K., Courville A., Salakhudinov R., Zemel R., Bengio Y. "Show, attend and tell: Neural image caption generation with visual attention." In International conference on machine learning 2015 Jun 1 (pp. 2048-2057).

3. Liu C., Mao J., Sha F., Yuille A. L. “Attention Correctness in Neural Image Captioning.” In AAAI 2017 Feb 4 (pp. 4176-4182).

4. You Q., Jin H., Wang Z., Fang C., Luo J. "Image captioning with semantic attention.” In Proceedings of the IEEE conference on computer vision and pattern recognition 2016 (pp. 4651-4659).

5. Zhao S., Sharma P., Levinboim T., \& Soricut R. "Informative Image Captioning with External Sources of Information," arXiv preprint arXiv:1906.08876, 2019.

6. See A., Liu P. J., \& Manning C. D. "Get to the point: Summarization with pointer-generator networks," arXiv preprint arXiv: 1704.04368, 2017.

7. Bahdanau D., Cho K., Bengio Y. "Neural machine translation by jointly learning to align and translate." arXiv preprint arXiv:1409.0473. 2014 Sep 1.

8. Luong M. T., Pham H., Manning C. D. "Effective approaches to attention-based neural machine translation". arXiv preprint arXiv:1508.04025. 2015 Aug 17.

9. Cheng J., Lapata M. "Neural summarization by extracting sentences and words." arXiv preprint arXiv:1603.07252. 2016 Mar 23

10. Zeng W., Luo W., Fidler S. \& Urtasun R. "Efficient summarization with read-again and copy mechanism," arXiv preprint arXiv:1611.03382, 2016.

11. Rush A. M., Chopra S., Weston J. "A neural attention model for abstractive sentence summarization.” arXiv preprint arXiv:1509.00685.2015 Sep 2.

12. Nallapati R., Zhai F., Zhou B. "Summarunner: A recurrent neural network based sequence model for extractive summarization of documents." In Thirty-First AAAI Conference on Artificial Intelligence 2017 Feb 12.

13. Vaswani A., et. al., I. Attention is all you need. In Advances in neural information processing systems, pp. 5998-6008, 2017.

14. Liu Y., \& Lapata M. "Hierarchical Transformers for Multi-Document Summarization," arXiv preprint arXiv:1905.13164, 2019

15. Carbonell J. G. \& Goldstein J. "The use of MMR, diversity-based reranking for reordering documents and producing summaries," In SIGIR, Vol. 98, pp. 335-336, 1998.

16. Fabbri A. R., Li I., She T., Li S., \& Radev D. R. "Multi-News: a Large-Scale Multi-Document Summarization Dataset and Abstractive Hierarchical Model. arXiv preprint arXiv:1906.01749, 2019.

17. Lebanoff L., Song K., \& Liu F. "Adapting the neural encoder-decoder framework from single to multi-document summarization," arXiv preprint arXiv:1808.06218, 2018.

18. Zhuge H. "Multi-Dimensional Summarization in Cyber-Physical Society," Morgan Kaufmann, 2016.

19. Mao J., Xu W., Yang Y., Wang J., Huang Z., Yuille A. "Deep captioning with multimodal recurrent neural networks (m-rnn)". arXiv preprint arXiv:1412.6632. 2014.

20. Jia X., Gavves E., Fernando B., Tuytelaars T. "Guiding the long-short term memory model for image caption generation." In Proceedings of the IEEE International Conference on Computer Vision 2015, pp. 2407-2415.

21. Wang C., Yang H., Bartz C., Meinel C. "Image captioning with deep bidirectional LSTMs." In Proceedings of the 2016 ACM on Multimedia Conference 2016, pp. 988-997.

22. Liu C., Sun F., Wang C., Wang F., Yuille A. "MAT: A multimodal attentive translator for image captioning." arXiv preprint arXiv:1702.05658, 2017.

23. Wang J., Madhyastha P., Specia L. "Object Counts! Bringing Explicit Detections Back into Image Captioning." arXiv preprint arXiv:1805.00314, 2018.

24. Venugopalan S., Anne Hendricks L., Rohrbach M., Mooney R., Darrell T., Saenko K. "Captioning images with diverse objects." In Proceedings of the IEEE Conference on Computer Vision and Pattern Recognition 2017, pp 5753-5761.

25. Gao, L., Fan, K., Song, J., Liu, X., Xu, X. and Shen, H.T., "Deliberate attention networks for image captioning," In Proceedings of the AAAI Conference on Artificial Intelligence, vol. 33, 2019, pp. 8320-8327.

26. Ren Z., Wang X., Zhang N., Lv X., Li L. J. "Deep reinforcement learning-based image captioning with embedding reward." arXiv preprint arXiv:1704.03899. 2017.

27. Chen C., Mu S., Xiao W., Ye Z., Wu L., Ma F., Ju Q. "Improving Image Captioning with Conditional Generative Adversarial Nets.” arXiv preprint arXiv:1805.07112. 2018.

28. Wang Q., Chan A. B. "CNN + CNN: Convolutional Decoders for Image Captioning." arXiv preprint arXiv:1805.09019, 2018. 
29. Simonyan K., Zisserman A. "Very deep convolutional networks for large-scale image recognition.” arXiv preprint arXiv:1409.1556, 2014

30. Krizhevsky A., Sutskever I., Hinton G. E. "Imagenet classification with deep convolutional neural networks.” In Advances in neural information processing systems, 2012, pp. 1097-1105.

31. Szegedy C., Liu W., Jia Y., Sermanet P., Reed S., Anguelov D., Erhan D., Vanhoucke V. A. "Rabinovich. Going deeper with convolutions." In Proceedings of the IEEE conference on computer vision and pattern recognition 2015, pp. 1-9.

32. He K., Zhang X., Ren S., Sun J. "Deep residual learning for image recognition.” In Proceedings of the IEEE conference on computer vision and pattern recognition, 2016, pp. 770-778.

33. Hu J., Shen L., Sun G. "Squeeze-and-excitation networks." In Proceedings of the IEEE Conference on Computer Vision and Pattern Recognition 2018, pp. 7132-7141.

34. Feng Y., Lapata M. "Automatic caption generation for news images." IEEE Trans. Pattern Anal. Mach. Intell. 35(4), 2013, pp.797-812.

35. Blei D. M., Ng A. Y., Jordan M. I. "Latent dirichlet allocation.” Journal of machine learning research. Vol. 3, 2003; pp.993-1022.

36. Batra V., He Y., Vogiatzis G. "Neural Caption Generation for News Images." In Proceedings of the Eleventh International Conference on Language Resources and Evaluation (LREC-2018), 2018.

37. Chen J., Zhuge H. "Abstractive Text-Image Summarization Using Multi-Modal Attentional Hierarchical RNN." In Proceedings of the 2018 Conference on Empirical Methods in Natural Language Processing 2018, pp. 4046-4056.

38. Chen, J., \& Zhuge, H. Extractive summarization of documents with images based on multi-modal RNN. Future Generation Computer Systems, 99, 2019, pp.186-196.

39. Sundermeyer, M., Schlüter, R., \& Ney, H. "LSTM neural networks for language modeling," In Thirteenth annual conference of the international speech communication association, 2012.

40. Cho K., Van Merriënboer B., Bahdanau D., Bengio Y. "On the properties of neural machine translation: Encoder-decoder approaches.” arXiv preprint arXiv:1409.1259, 2014.

41. Kingma D., Ba J. "Adam: a method for stochastic optimization". Computer Science. https://arxiv.org/pdf/1412.6980.pdf, 2014.

42. Hermann K. M., Kocisky T., Grefenstette E., Espeholt L., Kay W., Suleyman M., Blunsom P. 2015. "Teaching machines to read and comprehend.” In Advances in Neural Information Processing Systems, pp. 1693-1701.

43. Mikolov T., Sutskever I., Chen K., et al. "Distributed Representations of Words and Phrases and their Compositionality." 2013, 26:3111-3119.

44. Papineni K., Roukos S., Ward T., Zhu W. J. "Bleu: a Method for Automatic Evaluation of Machine Translation." In Proceedings of the Meeting of the Association for Computational Linguistics (ACL) 2002.

45. Denkowski M., Lavie A. "Meteor universal: Language specific translation evaluation for any target language," In Proceedings of the EACL 2014 Workshop on Statistical Machine Translation, 2014.

46. Lin C. Y. "Rouge: A package for automatic evaluation of summaries," Workshop on Text Summarization Branches Out, Association for Computational Linguistics, https:/www.aclweb.org/anthology/W04-1013, 2004.

47. Zhuge, H. "The Future Interconnection Environment," IEEE Computer, 38(4), 2005, pp.27-33.

48. Zhuge, H. "The Web Resource Space Model," Springer, 2008.

49. Zhuge, H. "Semantic Linking through Spaces for Cyber-Physical-Socio Intelligence: A Methodology," Artificial Intelligence, 175, 2011, pp.988-1019.

50. Zhuge, H. and Xing, Y. "Probabilistic Resource Space Model for Managing Resources in Cyber-Physical Society," IEEE Transactions on Service Computing, 5(3), 2012, pp404-421.

51. Zhuge, H. "The Knowledge Grid: Toward Cyber-Physical Society,” World Scientific Co. 2012.

52. Zhuge, H. "Cyber-Physical-Social Intelligence on Human-Machine-Nature Symbiosis," Springer, 2020.

53. Chen, J and Zhuge, H. "News image captioning based on text-summarization using image as query," The $15^{\text {th }}$ International Conference on Semantics, Knowledge and Grids (SKG2019), Guangzhou, China, 2019. 